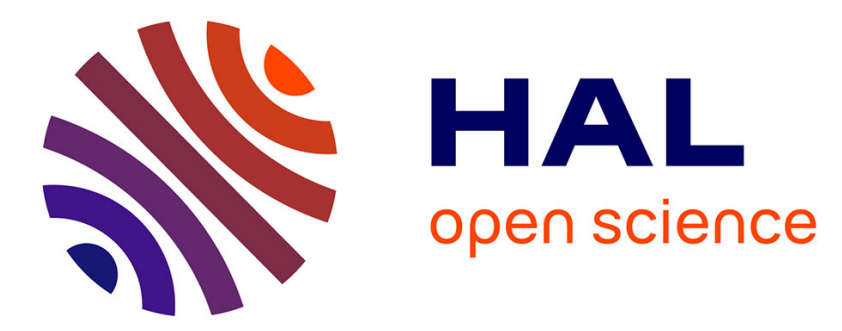

\title{
ANALYSE STATISTIQUE DES DONNÉES SPECTRORÉFLECTOMÉTRIQUES POUR L'ÉTUDE DES FILMS D'OXYDE
}

\author{
G. Blondeau, M. Froelicher, Michel Froment, Anne Hugot-Le Goff
}

\section{- To cite this version:}

G. Blondeau, M. Froelicher, Michel Froment, Anne Hugot-Le Goff. ANALYSE STATISTIQUE DES DONNÉES SPECTRORÉFLECTOMÉTRIQUES POUR L'ÉTUDE DES FILMS D'OXYDE. Journal de Physique Colloques, 1977, 38 (C5), pp.C5-157-C5-162. 10.1051/jphyscol:1977520 . jpa-00217169

\section{HAL Id: jpa-00217169 https://hal.science/jpa-00217169}

Submitted on 1 Jan 1977

HAL is a multi-disciplinary open access archive for the deposit and dissemination of scientific research documents, whether they are published or not. The documents may come from teaching and research institutions in France or abroad, or from public or private research centers.
L'archive ouverte pluridisciplinaire HAL, est destinée au dépôt et à la diffusion de documents scientifiques de niveau recherche, publiés ou non, émanant des établissements d'enseignement et de recherche français ou étrangers, des laboratoires publics ou privés. 


\title{
ANALYSE STATISTIQUE DES DONNÉES SPECTRORÉFLECTOMÉTRIQUES POUR L'ÉTUDE DES FILMS D'OXYDE
}

\author{
G. BLONDEAU, M. FROELICHER, M. FROMENT et A. HUGOT-LE GOFF \\ C. N. R. S. Physique des Liquides et Electrochimie (*), Université Pierre et Marie Curie \\ 4, Place Jussieu 75230 Paris cedex 05, France
}

\begin{abstract}
Résumé. - On décrit une méthode d'étude des films croissant sur un substrat métallique basée sur l'analyse statistique du pouvoir réflecteur $R$ des surfaces. Cette méthode est applicable à tous les cas d'oxydation, où se forment des films d'épaisseur supérieure à quelques dizaines d'Å. Elle consiste à déterminer les inconnues des équations de Fresnel par analyse statistique de $R$ en fonction de paramètres expérimentaux gouvernant l'épaisseur du film tels que durée ou température d'oxydation, tension anodique, etc... On peut ainsi, pour toute longueur d'onde s'étendant du proche $U$. V. à l'I. R., ajuster en même temps les indices $n$ et $k$ du film et certains paramètres inconnus du système. Dans un premier exemple, relatif à l'oxydation du nickel, on montre qu'il est possible d'ajuster en même temps que $n$ et $k$ les 2 paramètres décrivant la loi de croissance du film. Dans le second exemple, relatif à l'oxydation anodique du titane, on a ajusté simultanément les indices du film et ceux du titane sous-jacent.
\end{abstract}

\begin{abstract}
A method to study films growing on a metallic substrate is described. It is based on the statistical analysis of the reflectance $R$ of surfaces. This method can be applied to any oxidation experiment where films thicker than about $50 \AA$ are formed. One evaluates the unknowns of the Fresnel equations by statistical analysis of $R$ as function of experimental parameters linked to the film thickness such as temperature or time of oxidation, anodic voltage, etc... For any wave lengh, between near U. V. and I. R., it is possible to adjust at the same time $n$ and $k$ indices of the film and others unknown parameters of the system. In a first example, concerning oxidation of nickel, we show the possibility of adjusting at the same time $n$ and $k$ but also two parameters describing the film growth. In a second example, about oxidation of titanium, we have adjusted simultaneously the film and the substrate indices.
\end{abstract}

1. Introduction. - Des films cristallographiquement définis se forment à la surface d'une électrode dans certaines conditions électrochimiques ainsi, bien entendu, que par voie thermique. Dans des conditions électrochimiques d'oxydation, on distingue, de façon schématique, les films passifs, fins (quelques dizaines d'Å au maximum) et les films barrière apparaissant sur l'aluminium ou les éléments de transition des 4 premières colonnes, qui peuvent atteindre des épaisseurs considérables (plusieurs microns). On s'intéresse ici exclusivement aux films suffisamment épais pour être, d'un point de vue cristallographique, traités sans ambiguité comme un matériau massif.

Comment étudier de tels films sur le plan optique ? on peut naturellement, avoir recours aux techniques optiques classiques pour les couches minces ou d'adsorption : ellipsométrie, réflectance modulée. Il est cependant paradoxal de mettre en cuvre des techniques sophistiquées, alors que la simple modification du pouvoir réflecteur due à la présence du film transporte des informations qu'il suffirait de savoir traiter. Par ailleurs, on recherche des données de nature spectroscopique que l'ellipsométrie ne peut fournir que malaisément.

(*) Groupe de recherche associé no 4 .
Il ne faut pas cependant, tomber dans l'erreur inverse, c'est-à-dire modéliser les films barrière par exemple, de façon trop simpliste. Les considérer comme transparents (ce qui est souvent le cas dans la littérature) c'est oublier que les imperfections du réseau cristallin liées au mode de croissance (électrolytique, ou même thermique) entraînent toujours une certaine conduction par défaut, donc une absorption : dans certains oxydes, comme $\mathrm{NiO}$, de faibles écarts à la stæchiométrie peuvent élever de plusieurs ordres de grandeur le coefficient d'absorption dans la zone d'énergie précédant l'absorption fondamentale. Non seulement cette absorption ne doit pas être négligée, mais elle est un moyen de connaissance des mécanismes à l'intérieur du film.

La méthode que nous avons développée répond parfaitement à ces exigences, ce que nous allons détailler maintenant.

- La gamme d'énergie intéressante pour l'étude optique des films s'étend de 1 à $6 \mathrm{eV}$ environ; cette gamme couvre en effet le proche U.V., où l'absorption fondamentale (qui se produit entre 4 et $5 \mathrm{eV}$ pour la plupart des oxydes des métaux de transition) donne des informations sur les perturbations subies par la structure de bande du composé ; le domaine visible, où se produit généralement l'absorption supplémentaire 
due aux écarts à la stœchiométrie; le proche I. R. où l'évaluation de la constante diélectrique permet d'estimer les polarisabilités des ions. On obtient donc dans cette gamme d'énergie un ensemble d'informations qui permettent de situer les différences présentées par les films anodiques, du fait de leur mode particulier de croissance, avec leurs homologues massif et stœchiométrique.

- L'utilisation d'un spectroréflectomètre classique permet de couvrir exactement cette gamme. Par ailleurs, et c'est un point sur lequel nous reviendrons, l'utilisation d'un spectroréflectomètre à sphère intégratrice permet de tester simultanément la rugosité de l'électrode.

Pour appliquer au pouvoir réflecteur un traitement par optimisation des inconnues, il s'agit de choisir judicieusement le paramètre par rapport à quoi ce pouvoir réflecteur sera dérivé ; $R$ est fonction des indices des trois milieux successifs ( $N$ et $K$ pour le substrat, $n$ et $k$ pour le film d'oxyde, $y$ pour le milieu ambiant généralement considéré comme transparent), de l'épaisseur $d \mathrm{du}$ film et de la longueur d'onde $\lambda$. Les variations de $R$ avec $\lambda$, qui sont celles que l'on obtient le plus directement sur le plan expérimental, ne sont malheureusement absolument pas exploitables, puisque un ajustement statistique de $R(\lambda)$ mettrait en cuvre les dérivées $\mathrm{d} N / \mathrm{d} \lambda, \mathrm{d} n / \mathrm{d} \lambda \ldots$ etc, qui représentent des inconnues supplémentaires. Le paramètre qui s'impose comme variable est donc l'épaisseur des films, dont on est facilement maître, aussi bien d'ailleurs dans un processus thermique que dans un processus électrolytique. Par conséquent, $d$ est écrit comme une fonction paramétrable $d(\alpha, \beta, \ldots, X), X$ pouvant être, soit le temps d'oxydation, soit la température, dans le cas d'une réaction thermique, soit la tension anodique dans le cas de l'oxydation électrolytique d'un métal valve-bref le paramètre le plus commode et le plus accessible que l'on puisse choisir pour gouverner $d$, et on effectue l'ajustement statistique à longueur d'onde fixe à partir de $R(X)$. A partir de là, la méthode que nous avons mise au point est très souple et peut s'adapter à toutes sortes de situations expérimentales, ex situ ou in situ, suivant le type de connaissance du système qu'à l'expérimentateur : on peut être amené à ajuster, en même temps que $n$ et $k$, soit $N$ et $K$, soit $\alpha, \beta \ldots$ comme on le verra dans les exemples que nous allons présenter.

2. Méthode expérimentale. - La figure 1 présente le montage utilisé pour suivre une oxydation anodique : une cellule électrolytique est adaptée aux fenêtres de la

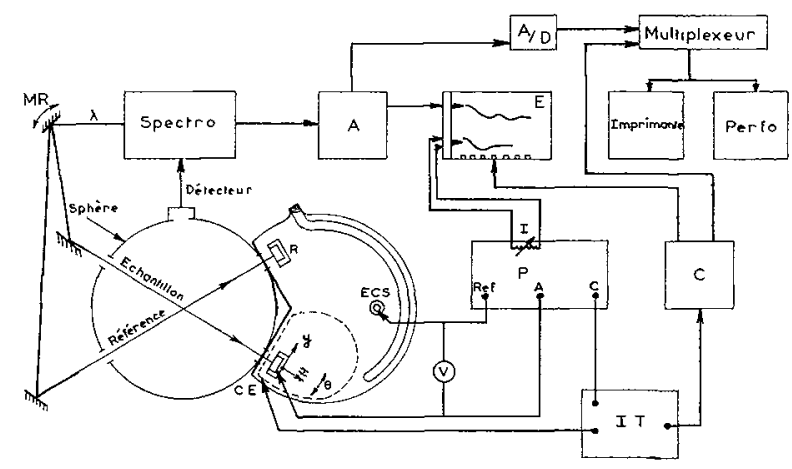

FIG. 1. - Synoptique du montage pour les études spectroréflectométriques in situ.

sphère intégratrice du spectroréflectomètre ; le déplacement micrométrique de l'échantillon permet d'égaliser les trajets optiques, entre la voie échantillon et la voie référence optique qui est généralement constituée par du platine; le signal analogique $R$ du spectroréflectomètre est converti en signal digital; dans le cas de la figure, c'est la quantité d'électricité $Q$ ayant traversé la cellule qui représente le paramètre variable $X$ : un dispositif électronique permet d'optimiser le nombre de points pris en compte en les ressérant plus ou moins selon la plus ou moins rapide variation de la fonction $R(Q)$. Finalement, un multiplexeur commande le perforateur de ruban de manière à ce que les informations puissent être directement traitées par ordinateur [1]. C'est le montage décrit ici qui a été en particulier mis en œuvre dans le premier exemple que nous allons présenter

3. Méthode de calcul. - Le pouvoir réflecteur $R$ est donné par $R=\rho \rho^{*}$

$$
\rho=\exp \left[4 \pi_{j} v d(\alpha \ldots X) / \lambda\right] \frac{(\tilde{n}+\tilde{N})(v-\tilde{n}) \exp A+(\tilde{n}-\tilde{N})(v+\tilde{N}) \exp -A}{(\tilde{n}+\tilde{N})(v+\tilde{n}) \exp A+(\tilde{n}-\tilde{N})(v-\tilde{n}) \exp -A}
$$

avec

$$
A=2 \pi_{j} \tilde{n} d(\alpha \ldots X) / \lambda .
$$

L'ajustement des résultats expérimentaux à cette expression théorique se fait en utilisant la méthode des moindres carrés généralisée, c'est-à-dire en minimisant [2] $\mathbf{S}^{\prime 2}=(\mathbf{E}-\widehat{\mathbf{E}})^{\prime} \mathbf{V}^{-1}\left(\mathbf{E}-\mathbf{E}^{\prime}\right)$, où $\mathbf{E}$ est le vecteur d'observations (un ensemble ordonné des valeurs mesurées de $R$ ), $\widehat{\mathbf{E}}$ est le vecteur des valeurs théoriques correspondant à $R$ observé, $\mathbf{V}$ est le vecteur d'erreur (chaque élément de $\mathbf{V}$ est le produit de l'erreur dans la mesure de $R$ et de l'erreur dans la mesure de $X$ ), l'apostrophe désigne une transposition de matrice. Cette méthode a été considérablement perfectionnée grâce au traitement de Tournarie [3], qui ajoute une connaissance a priori des paramètres. La méthode de Nelder et Mead [4] permet une minimisation plus facile. Un programme FORTRAN mis au point au labora. 
toire donne les paramètres ajustables avec leurs intervalles de confiance et leurs erreurs-type [5].

4. Ajustement sur 4 paramètres (in situ) $: n, k, \alpha, \beta$. L'exemple présenté ici est celui de l'oxydation anodique du nickel en solution sulfurique concentrée. Lorsque la concentration en acide augmente (elle est de $14 \mathrm{~N}$ dans le cas des résultats présentés figure 2),

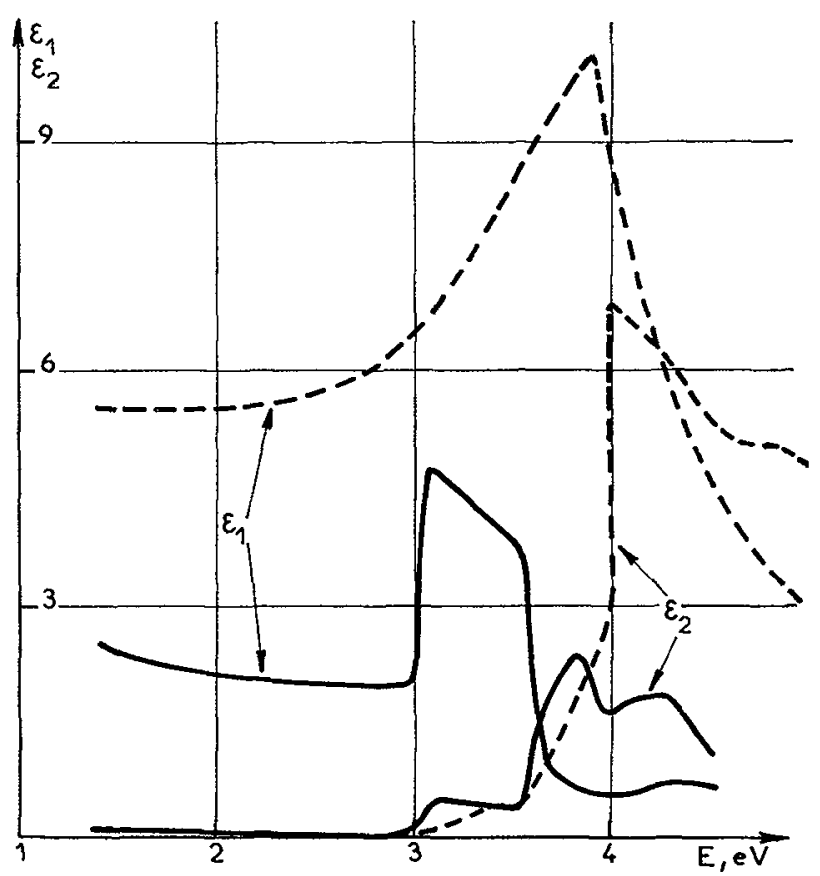

FIG. 2. - Parties réelle $\varepsilon_{1}$ et imaginaire $\varepsilon_{2}$ de la constante diélectrique des films anodiques formés sur le nickel.

---- Valeurs relatives à $\mathrm{NiO}$ massif [7].

le film passif de $\mathrm{NiO}$ [6] (présent entre 500 et $1000 \mathrm{mV} /$ ECS dans les solutions faiblement concentrées) se trouve dégradé par l'introduction d'atomes de soufre dans le réseau cristallin ; les défauts permettent alors une importante conduction ionique et le film croit, pouvant atteindre une épaisseur très élevée - n'ayant plus rien de commun avec les couches passives: $2750 \AA$ à $750 \mathrm{mV} / \mathrm{ECS}$. Dans ce cas, la diffraction électronique confirme que le réseau cristallin de $\mathrm{NiO}$ cfc (paramètre 4,176 Å est rigoureusement conservé); mais le film est poreux (les pores sont visibles en microscopie électronique à haute résolution), et contient d'importantes quantités de soufre. Un ensemble de mesures, dosages radiochimiques de soufre, comptage d'oxygène par observation directe de réactions nucléaires, déterminations directes de densité, confirment que la composition stœchiométrique assez singulière de ces films se rapprocherait de $\mathrm{NiO}_{1,4} \mathrm{~S}_{0,2}, 40 \% \mathrm{du}$ volume environ étant occupé par les pores. On peut donc se demander dans quelle mesure le comportement optique d'un tel oxyde a encore quelque chose de commun avec celui de NiO.

En fait, de façon paradoxale, ce système électrochimique qui nous a antérieurement permis de mettre au point et de tester le montage d'études in situ n'est pas le mieux adapté que l'on puisse imaginer, et.cela pour deux raisons. La première est qu'une condition essentielle pour la validité du calcul est que les indices optiques du film restent invariants lorsque l'on se déplace d'un interface à l'autre : $n$ et $k$ doivent être indépendants de $d$. Or il semble qu'il y ait un gradient de concentration, aussi bien en ce qui concerne le nickel que l'oxygène, du métal à la solution. Dans ce cas le calcul va donner une valeur moyennée des indices; nous reviendrons ultérieurement sur ce point, car cette difficulté peut effectivement être tournée. Par ailleurs, ce système échappant à tout modèle d'oxydation classique, on ne peut utiliser une loi de croissance connue, du type Mott-Cabrera par exemple. On a dû tester, de manière parfaitement empirique diverses écritures par la loi de croissance du film. Celle-ci résultant d'un double mécanisme de formation à l'interface métal-film et de dissolution à l'interface film-électrolyte, on a essayé (sans succès) en particulier des différences d'exponentielles. En fin de compte, une loi de croissance ne faisant intervenir que deux paramètres ajustables $d=\alpha \tanh (\beta Q)$ a donné satisfaction. $\alpha$ représente $d_{1 i m}$, épaisseur limite atteinte par le film au bout d'un certain temps d'oxydation.

Finalement, le seul intérêt du système est la lenteur des processus (l'épaisseur limite n'est atteinte qu'en une dizaine de minutes), qui nous a permis de travailler sur un nombre élevé de couples $R, Q$, bien que nous ayons utilisé un spectroréflectomètre assez ancien, à transmission mécanique et temps de réponse long: un spectroréflectomètre d'une nouvelle génération, suivie d'une électronique adaptée pour la liaison avec la perforatrice, doit permettre d'étudier sans difficulté des systèmes beaucoup plus rapides. Dans le cas présent, chaque point a été obtenu par un calcul mettant en jeu 80 couples $R, Q$ environ. A chaque longueur d'onde désirée l'oxydation est donc répétée avec une électrode différente. A titre d'exemple, les courbes de la figure 2 ont nécessité la réalisation de 65 électrodes, ce qui correspond à un pas de $0,0125 \mu \mathrm{m}$ entre deux déterminations expérimentales. Bien entendu, le calcul doit nous fournir, pour chaque longueur d'onde des valeurs différentes de $n$ et $k$ mais les mêmes valeurs de $d_{1 \text { im }}$ et $\beta$ qui sont caractéristiques des conditions électrochimiques du système exclusivement : on a donc là une parfaite vérification a posteriori de la validité de la méthode. En pratique, on constate que les électrodes préparées dans des conditions électrochimiques strictement identiques se recouvrent de films dont l'épaisseur manifeste une certaine dispersion. On est donc contraint de tolérer quelques \% d'irréproductibilité dans les résultats du calcul : une valeur moyenne $d=2750 \tanh (0,50 Q)$ se dégageant, tous les échantillons pour lesquels $d_{\text {lim }}$ ou $\beta$ s'écartent de plus de $5 \%$ des valeurs moyennes sont rejetés. Dans un deuxième temps nous avons vérifié que les valeurs de $n$ et $k$ obtenues dans un autre calcul, où $d_{1 \mathrm{im}}$ est fixé à $2750 \AA$ et $\beta$ à 0,50 restent comparables aux pré- 
cédentes, toujours bien entendu dans la limite de quelques $\%$ compatibles avec le système électrochimique.

Les propriétés optiques des films anodiques sont comparées à celles de $\mathrm{NiO}$ massif et stœchiométrique, calculées par analyse de Kramers-Kronig [7] (ici les deux courbes se trouvent considérablement agrandies par rapport aux originaux, la méthode de KramersKronig n'étant évidemment pas adaptée à fournir une telle précision). On a tracé $\varepsilon_{1}=n^{2}-k^{2}$ et $\varepsilon_{2}=2 n k ;$ malgré la profonde perturbation du réseau, on retrouve un certain nombre de caractéristiques communes aux deux spectres : l'adsorption fondamentale est décalée vers les plus faibles énergies, de $0,4 \mathrm{eV}$ environ. Les deux pics sont centrés à $3,8 \mathrm{eV}$ et $4,3 \mathrm{eV}$ au lieu de 4 et 4,8 eV. L'absorption est beaucoup moins intense, c'est-à-dire qu'un nombre réduit de porteurs de charge est mis en jeu. Du côté I. R. du spectre, la très faible valeur de $\varepsilon_{1}$ est en relation directe avec la nature poreuse du composé. En fait, c'est entre 3 et $3,4 \mathrm{eV}$ environ que le comportement optique des oxydes anodiques est étranger à celui de $\mathrm{NiO}$ : dans cette gamme d'énergie où ne devraient exister que des transitions de champ cristallin, qui ne sont pas décelables à l'échelle de la présente figure et que nous avons pu étudier par ailleurs à l'aide de notre méthode sur des oxydes de nickel observés ex situ [8], on met en évidence une intense bande d'absorption centrée à $3,1 \mathrm{eV}$ et intervenant particulièrement dans la partie réelle de la constante diélectrique.

Il est inutile d'insister plus sur l'interprétation des résultats ; l'intérêt de l'exemple était de montrer qu'il est possible de décrire totalement un phénomène d'oxydation (indices optiques du film dans un large domaine de longueurs d'onde, loi de croissance et épaisseur d'équilibre) en n'ayant au départ qu'une connaissance très médiocre du système, et en mettant en œuvre une technologie relativement simple. L'établissement du programme de calcul est la partie la plus lourde de la méthode. Un cas d'oxydation thermique aurait, évidemment, illustré la méthode de façon plus agréable.

5. Ajustement sur 4 paramètres (ex situ) : $n, k, N, K$. — Ce problème s'est posé à nous à propos de l'oxydation anodique du titane en solution sulfurique. Dans ce cas, une relation linéaire $d=\alpha V$ relie l'épaisseur du film à la tension anodique, et nous avons déterminé $\alpha=25 \AA / \mathrm{V}$ [9]. Les films présentent deux structures différentes, suivant que la tension d'oxydation est supérieure ou inférieure à $50 \mathrm{~V}$. Les films les plus épais sont formés d'anatase $\mathrm{TiO}_{2}$; les films les plus fins, de microcristallites contenant de 20 à 25 atomes arrangés avec les symétries de l'anatase [10], ce que nous avons pu mettre en évidence par diffraction électronique et simulations.

Notre but a donc été de mettre en évidence les modifications de propriétés optiques liées à la distance de l'ordre structural. Le problème principal s'est trouvé posé par la mauvaise connaissance des indices optiques du substrat titane : ceux-ci peuvent présenter des écarts considérables en fonction de la nature du métal, des inclusions qu'il peut contenir et de son traitement de surface. La solution raisonnable consistait donc à les ajuster en même temps que $n$ et $k$, puisque par ailleurs la loi de croissance était bien connue, la tension anodique $V$ jouant ici le rôle du paramètre variable $X$. Ici on a travaillé $e x$ situ, sur un ensemble discret de couples $R, V$; on constitue un tel ensemble en oxydant des électrodes de titane à des tensions réparties de façon régulière, par exemple tous les volts, ou tous les deux volts, et en se plaçant ensuite à une longueur d'onde déterminée comme le montre la figure 3. Nous avons constaté que la précision de l'ajustement dépen-

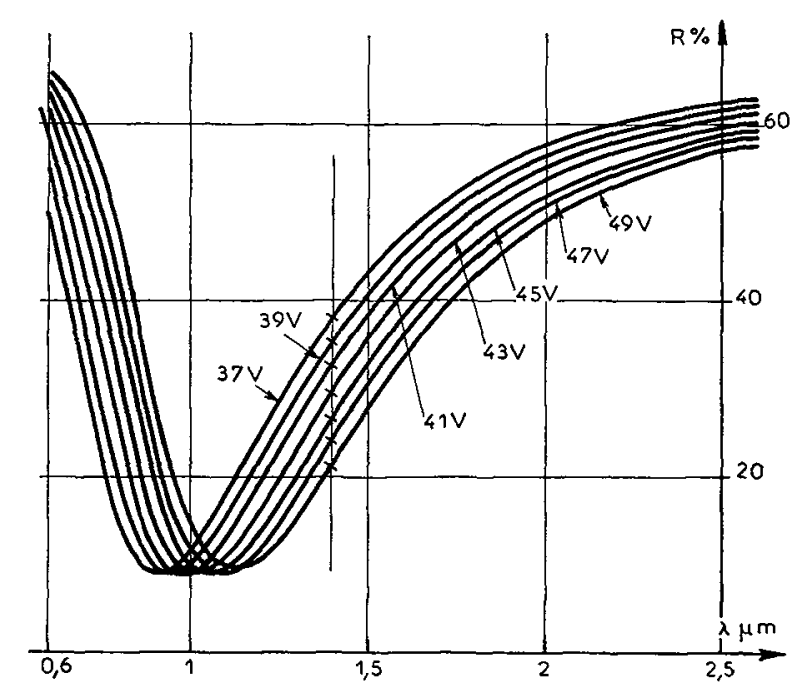

Fig. 3. - Pouvoirs réflecteurs d'un ensemble d'électrodes de titane oxydées tous les deux volts.

dait directement du rapport entre l'épaisseur moyenne des films dans l'ensemble considéré, et la longueur d'onde. Ainsi, pour un ensemble de films formés d'anatase - ayant donc des épaisseurs élevées $N$ et $K$ (ainsi que $n$ et $k$ bien entendu) étaient obtenus avec de très bons intervalles de confiance pour des longueurs d'onde supérieures à $0,7 \mu \mathrm{m}$ environ, et avec des intervalles de confiance inacceptables en dessous de cette longueur d'onde. Au contraire, pour un ensemble de films microcristallins - donc de faible épaisseur, $N$ et $K$ étaient obtenus avec de très bons intervalles de confiance en dessous de $0,7 \mu \mathrm{m}$, et présentaient aux longueurs d'onde élevées des valeurs erratiques reproduisant d'assez loin les valeurs de Johnson et Christy [11] nous ayant servi à initialiser le calcul. Entre 0,5 et $1 \mu \mathrm{m}$ environ, les deux calculs conduisaient à des valeurs identiques de $N$ et $K$ [12].

En effet, un calcul fait à longueur d'onde élevée sur un ensemble d'épaisseurs faibles met en jeu des changements du pouvoir réflecteur du même ordre que l'erreur expérimentale dans la détermination de $R$ et le calcul perd toute précision. Inversement, un calcul fait à 
faible longueur d'onde sur un ensemble d'épaisseurs élevées met en jeu des variations $\mathrm{d} R / \mathrm{d} V$ très élevées, et le calcul devient très difficile.

Par conséquent, en rassemblant les résultats des deux calculs (en pratique nous avons testé un nombre plus élevé d'ensembles) nous avons pu, comme le montre la figure 4, obtenir $N$ et $K$ dans la totalité du

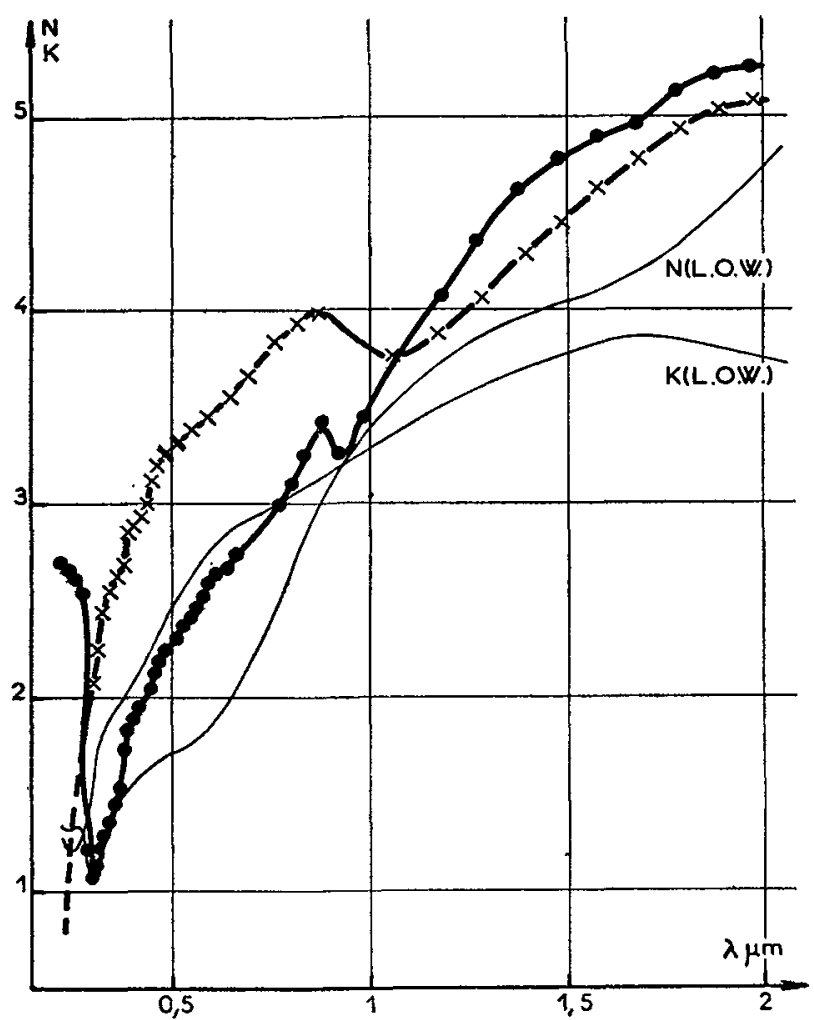

FIG. 4. - Indices optiques $N$ et $K$ du titane déterminés par la méthode spectroréflectométrique.

spectre. Nous avons pu alors constater un excellent accord avec des valeurs plus récentes données par Lynch et coll. [13] (notées LOW sur la figure), non pas en ce qui concerne les valeurs absolues, ce qui n'aurait aucun sens puisque ces dernières valeurs ont été obtenues par analyse de Kramers-Kronig mais en ce qui concerne l'allure générale des variations de $N$ et $K$ et les positions des points caractéristiques comme la fréquence de plasmons à $0,27 \mu \mathrm{m}$.

Etant donc en possession des bonnes valeurs de $N$ et $K$, ou du moins des valeurs intervenant effectivement dans nos expériences, nous avons pu les introduire dans un calcul ultérieur, de manière à obtenir les propriétés optiques des films de $\mathrm{TiO}_{2}$. La figure 5 présente les valeurs de $\varepsilon_{1}$ et $\varepsilon_{2}$ pour l'oxyde microcristallin : on met en évidence un comportement parfaitement lorentzien ; l'absorption fondamentale se présente comme une bande large, que l'on peut déconvoluer en faisant intervenir deux oscillateurs, en relation avec le dédoublement du niveau $3 \mathrm{~d} d \mathfrak{d}$ titane par le champ cristallin. Les deux bandes sont alors centrées à 3,83 et $4,20 \mathrm{eV}$.

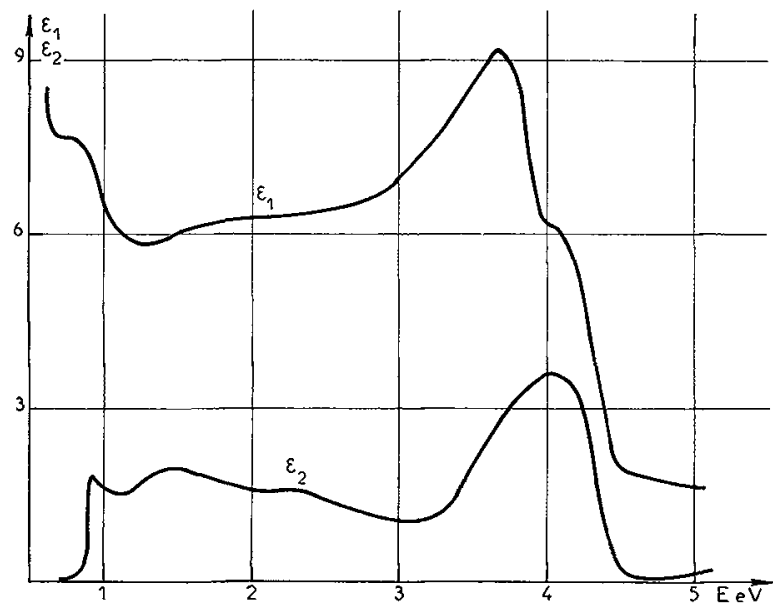

FIg. 5. - Parties réelle $\varepsilon_{1}$ et imaginaire $\varepsilon_{2}$ de la constante diélectrique des films microcristallins de $\mathrm{TiO}_{2}$.

Dans le domaine visible, on constate une forte absorption que l'on peut attribuer à la présence de vacances d'oxygène associées à des ions $\mathrm{Ti}^{3+}$. En effet, la très petite taille des cristallites entraîne nécessairement qu'une proportion notable du volume est constituée de joints de grains, et donc de sites qui ne sont pas occupés de façon normale. Nous n'insisterons pas plus sur ces résultats, les comparaisons avec les propriétés optiques des films d'anatase ayant été discutées par ailleurs [14].

Avant de terminer, il convient de revenir sur deux points. Le premier est relatif à une extension possible de la méthode. Il a été dit en effet que l'emploi de celle-ci était limité exclusivement par la condition de constance des indices du film dans son épaisseur. C'est une exigence commune à toutes les mesures optiques mais que nous avons en fait la possibilité de surmonter. Si les indices du substrat ou la loi de croissance du film ne posent pas de problème spécifique, il est possible d'écrire $n$ et $k$ comme des fonctions de l'épaisseur. En choisissant de simples fonctions linéaires, on est ramené à résoudre un problème d'ajustement sur 4 paramètres, ce qui est parfaitement abordable comme nous venons de le montrer. Cette approximation linéaire est un progrès appréciable pour les films présentant un gradient dans la concentration de certains éléments, comme les films formés sur alliage par exemple. Bien entendu, le problème de l'oxydation du nickel présenté ici était beaucoup trop complexe pour être redevable de ce mode de calcul, mais on peut imaginer de nombreux cas favorables.

Enfin, grâce à cet appareillage aussi banal à première vue qu'un spectroréflectomètre à sphère intégratrice, on peut parallèlement aux études d'oxydation, contrôler constamment la rugosité de l'électrode. Le rôle de la rugosité dans les propriétés optiques d'un interface revient constamment en tant que question dans les préoccupations des électrochimistes, d'autant qu'en ellipsométrie par exemple, il est très difficile de séparer son influence de celle d'un film. On voit trop 
souvent intervenir un facteur de rugosité dont la signification exacte n'est pas claire, car sa valeur est trop élevée pour représenter réellement le rapport de la surface effective de l'électrode, à la surface de base. Dans nos études, nous enregistrons, en même temps que le pouvoir réflecteur de l'électrode, l'évolution avec la longueur d'onde de l'énergie lumineuse diffractée en dehors de la direction spéculaire, $R_{\mathrm{d}}$. Le rapport de $R_{\mathrm{d}}$ à $R$ permet d'estimer la rugosité de la surface, ou même dans un grand nombre de cas de la calculer avec précision, par l'intermédiaire de sa dénivellation quadratique moyenne, $\sigma$. Si $\sigma$ est petit devant $\lambda$, ce qui est généralement le cas après un traitement électro- chimique classique, on peut écrire au premier ordre $R_{\mathrm{d}} / R=4 \Pi \sigma / \lambda^{2}$. Avec un spectroréflectomètre tel que celui que nous utilisons, on ne peut pas calculer $\sigma$ sur des surfaces caractérisées par une distance d'autocorrélation entre défauts trop élevée, mais de tels cas se présentent rarement dans les systèmes que nous étudions [15]. Donc, étant en mesure de contrôler la rugosité des surfaces, même pour de faibles valeurs de celle-ci (on peut mesurer $\sigma$ compris entre $15 \AA$ et $1000 \AA$ environ) nous sommes en mesure aussi, soit d'affirmer que son influence est négligeable, soit d'orienter nos efforts vers une amélioration de l'état géométrique de l'interface.

\section{Bibliographie}

[1] Blondeau, G., Froelicher, M., Froment, M. et HugotLe GoFf, A., Proc. Symp. Oxide-Electrolyte Interfaces, Miami Beach (1972), R. Alwitt, ed. (Electrochemical Society Inc, Princeton) 1973, 215.

[2] Kendall, M. 'G. et Stuart, A., The Advanced Theory of Statistics, Vol. 2, Griffin (London) 1967, p. 75.

[3] Tournarie, M., J. Physique 30 (1969) 737.

[4] Nelder, J. A. et MeAd, R., Comput. J. 7 (1965) 308.

[5] Badiali, J. P., Cachet, H. et Lestrade, J. C., J. Chem. Soc., Faraday Trans. 2 (1973) 1339.

[6] Siejka, J., Cherkx, C., Yahalom, J., J. Electrochem. Soc. 119 (1972) 991.

[7] Powell, R. J. et Spicer, W. E., Phys. Rev, B 2 (1970), 2182.

[8] Blondeau, G., Froelicher, M., Froment, M. et HugotLe Goff, A., Phys. Status Solidi (a) 26 (1974) 181.
[9] Arsov, Lj., Froblicher, M., Froment, M. et HugotLe Goff, A., J. Chim. Phys. 72 (1974) 275.

[10] Blondeau, G., Froelrcher, M., Froment, M., HugotLe Gofe, A., Brieu, M., Calsou, R. et Larroque, P., J. Microsc. Spectrosc. Electron. 2 (1977) 27.

[11] Christy, R. W. et Johnson, P. B., Phys. Rev. B 9 (1974) 5056.

[12] Blondeau, G., Froelicher, M., Froment, M. et HugotLe Goff, A., Thin Solid Films 38 (1976) 261.

[13] Lynch, D. W., Olson, C. G. et Weaver, J. H., Phys. Rev. B 11 (1975) 3617.

[14] Blondeau, G., Froelicher, M., Froment, M. et HugotLe GoFf, A., Thin Solid Films 42 (1977) 147.

[15] Hugot-Le GoFf, A., Galvanotech. Oberflächenschutz 8 (1967), 97, 121 et 143. 